TRANSACTIONS OF THE

AMERICAN MATHEMATICAL SOCIETY

Volume 349, Number 1, January 1997, Pages 217-233

S 0002-9947(97)01754-6

\title{
ESSENTIALLY NORMAL OPERATOR + COMPACT OPERATOR $=$ STRONGLY IRREDUCIBLE OPERATOR
}

\author{
CHUNLAN JIANG, SHUNHUA SUN, AND ZONGYAO WANG
}

\begin{abstract}
It is shown that given an essentially normal operator $T$ with connected spectrum, there exists a compact operator $K$ such that $T+K$ is strongly irreducible.
\end{abstract}

\section{INTRODUCTION}

Let $\mathcal{L}(\mathcal{H})$ denote the algebra of all bounded linear operators acting on a complex, separable, infinite dimensional Hilbert space $\mathcal{H}$. An operator $T \in \mathcal{L}(\mathcal{H})$ is said to be strongly irreducible if it does not commute with any nontrivial idempotent [10], [15]. The strong irreducibility is invariant under similarity. Chun-lan Jiang and Zong-yao Wang proved that for each operator $T$ with connected spectrum $\sigma(T)$, there exists a strongly irreducible operator $A$ and a sequence $\left\{X_{n}\right\}$ of invertible operators such that $\left\|X_{n} A X_{n}^{-1}-T\right\| \rightarrow 0(n \rightarrow \infty)$ [13]. This result strengthened the following result of D. A. Herrero and Chun-lan Jiang: The norm closure of the set of strongly irreducible operators $=$ the set of the operators with connected spectra.

An operator $T \in \mathcal{L}(\mathcal{H})$ is irreducible if it does not commute with any nontrivial orthogonal projection. P. R. Halmos proved that for each $T \in \mathcal{L}(\mathcal{H})$ and $\epsilon>0$, there exists a $K$ compact with $\|K\|<\epsilon$ such that $T+K$ is irreducible [11]. It is obvious that if $\sigma(T)$ is not connected, then for each $K$ compact, $T+K$ is still strongly reducible. Thus D. A. Herrero asked the following questions in personal communications:

1. Given $T \in \mathcal{L}(\mathcal{H})$ with connected $\sigma(T)$, does there exist a compact operator $K$ such that $T+K$ is strongly irreducible?

2. Given an essentially normal operator $T$ with connected $\sigma(T)$, does there exist a compact operator $K$ such that $T+K$ is strongly irreducible? An operator $T$ is essentially normal if $T^{*} T-T T^{*}$ is compact, where $T^{*}$ denotes the dual of $T$.

This article proves that the second question has an affirmative answer.

Main Theorem. Given an essentially normal operator $T$ with connected spectrum, there exists a compact operator $K$ such that $T+K$ is strongly irreducible.

Received by the editors May 25, 1995.

1991 Mathematics Subject Classification. Primary 47A10, 47A55, 47A58.

Key words and phrases. Essentially normal operator, strongly irreducible operator, Sobolev space, Cowen-Douglas operator, subnormal operator, Rosenblum operator.

The research supported by National Natural Science Foundation of China. 
If we can construct a strongly irreducible essentially normal operator $S$ such that $\sigma_{e}(S)=\sigma_{e}(T)$ and $\operatorname{ind}(\lambda-S)=\operatorname{ind}(\lambda-T)$ for all $\lambda \notin \sigma_{e}(S)$, then by Brown-DouglasFillmore Theorem $S$ is unitarily equivalent to some compact perturbation $T+K$ of $T$. Thus, the proof of the main theorem is reduced to constructing a strongly irreducible essentially normal operator with the right spectral picture. A more interesting question is if we can manage to obtain a small compact perturbation in the main theorem. Here the Brown-Douglas-Fillmore Theorem does not work. We have to develop more special techniques. In another paper, the authors and Y. Q. Ji have obtained the following result.

Theorem (J-J-W). Given an essentially normal operator $T$ with connected spectrum. If $\operatorname{ind}(\lambda I-T)>0$ for all $\lambda \in \rho_{F}(T)$, then for each $\epsilon>0$, there exists $K$ compact with $\|K\|<\epsilon$ such that $T+K$ is strongly irreducible.

\section{Preparation}

In what follows, $T \in(\mathrm{SI})$ means that $T$ is a strongly irreducible operator on its acting space and $\Omega$ always denotes a bounded, connected open set in $C$. Recall that for natural number $n, \mathcal{B}_{n}(\Omega)$, the set of Cowen-Douglas operators of index $n$, is the set of all operators $B$ on $\mathcal{H}$ satisfying

(i) $\sigma(B) \supset \Omega$;

(ii) $\operatorname{nul}(\lambda-B)=\operatorname{ind}(\lambda-B)=n(\lambda \in \Omega)$;

(iii) $\bigvee\{\operatorname{ker}(\lambda-B): \lambda \in \Omega\}=\mathcal{H}[5],[6]$;

Note that (iii) can be replaced by (iii)' [6]:

(iii)' $\bigvee\left\{\operatorname{ker}\left(\lambda_{0}-B\right)^{k}: k=1,2, \cdots\right\}=\mathcal{H}$ for each $\lambda_{0} \in \Omega$.

Also note that each operator $B$ in $\mathcal{B}_{1}(\Omega)$ is strongly irreducible [6], and recall that if $N(\Omega)$ is the "multiplication by $\lambda$ " operator acting on $L^{2}(\Omega)$ and $N_{+}(\Omega)$, the Bergman operator, is the restriction of $N(\Omega)$ to $L_{a}^{2}(\Omega)$, the subspace of $L^{2}(\Omega)$ spanned by the rational functions with poles outside $\bar{\Omega}$, then

(i) $\mathcal{A}^{\prime}\left(N_{+}(\Omega)\right)=\mathcal{H}^{\infty}(\Omega)[12]$ and $N_{+}(\Omega)$ is subnormal and essentially normal, where $\mathcal{A}^{\prime}\left(N_{+}(\Omega)\right)$ denotes the commutant of $N_{+}(\Omega)$;

(ii) If $N=N_{+}\left(\Omega^{*}\right)^{*}$, then $N \in \mathcal{B}_{1}(\Omega), \sigma_{l}(N)=\sigma(N)=\bar{\Omega}, \sigma_{e}(N)=\sigma_{r}(N)=\partial \bar{\Omega}$, where $\Omega^{*}=\{\lambda \in C: \bar{\lambda} \in \Omega\}$ and $\partial \bar{\Omega}$ denotes the boundary of $\bar{\Omega}$.

Given $A, B \in \mathcal{L}(\mathcal{H})$, the Rosenblum operator $\tau_{A B} \in \mathcal{L}(\mathcal{L}(\mathcal{H}))$ is defined by $\tau_{A B}(X)=A X-X B$. It is well known that

(i) $\sigma\left(\tau_{A B}\right)=\sigma(A)-\sigma(B)$;

(ii) $\tau_{A B}$ is surjective if and only if $\sigma_{r}(A) \cap \sigma_{l}(B)=\varnothing[12]$.

Lemma 2.1 ([13, Lemma 2]). Let $A, B \in \mathcal{L}(\mathcal{H})$. Assume that

$$
\mathcal{H}=\bigvee\left\{\operatorname{ker}(\lambda-B)^{k}: \lambda \in \Gamma, k \geq 1\right\}
$$

for certain subset $\Gamma$ of the point spectrum $\sigma_{p}(B)$ of $B$ and $\sigma_{p}(A) \cap \Gamma=\varnothing$; then $\operatorname{ker} \tau_{A B}=\{0\}$. 
Lemma 2.2. Given $A \in \mathcal{B}_{1}(\Omega)$ and $\lambda_{0} \in \Omega$, then there exist an $O N B\left\{e_{n}\right\}_{n=1}^{\infty}$ of $\mathcal{H}$ and $r>0$ such that

$$
A=\left(\begin{array}{cccc|c}
\lambda_{0} & a_{12} & a_{13} & \ldots \\
& \lambda_{0} & a_{23} & \ldots & e_{1} \\
& & \lambda_{0} & \ldots \\
0 & & & \ldots
\end{array}\right) e_{2}
$$

and $\left|a_{k, k+1}\right|>r>0(k=1,2, \cdots)$.

Proof. Assume that $e_{1} \in \operatorname{ker}\left(A-\lambda_{0}\right)$ and $\left\|e_{1}\right\|=1$. Let $B$ be the right inverse of $A-\lambda_{0}$. Since $\operatorname{ker} B^{k}=\{0\}(k=1,2, \cdots)$ and $e_{1} \notin \operatorname{ran} B,\left\{e_{1}, B e_{1}, B^{2} e_{1}, \cdots\right\}$ is linearly independent. Since $B^{k-1} e_{1} \in \operatorname{ker}\left(A-\lambda_{0}\right)^{k}$ and $\operatorname{nul}\left(A-\lambda_{0}\right)^{k}$ $=k, \operatorname{ker}\left(A-\lambda_{0}\right)^{k}=\bigvee\left\{e_{1}, B e_{1}, B^{2} e_{1}, \cdots, B^{k-1} e_{1}\right\} \quad(k=1,2, \cdots)$. Since $\bigvee\left\{\operatorname{ker}\left(A-\lambda_{0}\right)^{k}, k=1,2, \cdots\right\}=\mathcal{H}, \bigvee\left\{B^{k} e_{1}: k=0,1,2, \cdots\right\}=\mathcal{H}$. Let $\left\{e_{k}\right\}_{k=1}^{\infty}$ be the Gram-Schmidt orthonormalization of $\left\{B^{k} e_{1}\right\}_{k=0}^{\infty}$. Then $A$ has an upper triangular matrix representation

$$
A=\left(\begin{array}{cccc}
\lambda_{0} & a_{12} & a_{13} & \ldots \\
& \lambda_{0} & a_{23} & \ldots \\
& & \lambda_{0} & \ldots \\
0 & & & \ldots
\end{array}\right)
$$

with respect to the ONB $\left\{e_{k}\right\}_{k=1}^{\infty}$. Note that $A-\lambda_{0}$ is bounded from below; thus there exists $r>0$ such that $\left\|\left(A-\lambda_{0}\right) y\right\| \geq r\|y\|$ for each $y \in\left[e_{1}\right]^{\perp}$. Set $x_{k}=a_{k, k+1} e_{k}$ and $x_{k}^{1}=-\sum_{i=1}^{k-1} a_{i, k+1} e_{i}(k=1,2, \cdots)$. Since $A-\lambda_{0}$ is onto, there is a vector $y_{k} \in \bigvee\left\{e_{i}: i=2,3, \cdots, k\right\}$ such that $x_{k}^{1}=\left(A-\lambda_{0}\right) y_{k}$. Thus $x_{k}=\left(A-\lambda_{0}\right)\left(e_{k+1}+y_{k}\right)$ and

$$
\begin{aligned}
\left|a_{k, k+1}\right|=\left\|x_{k}\right\| & =\left\|\left(A-\lambda_{0}\right)\left(e_{k+1}+y_{k}\right)\right\| \\
& \geq r\left\|e_{k+1}+y_{k}\right\| \\
& =r \sqrt{\left\|e_{k+1}\right\|^{2}+\left\|y_{k}\right\|^{2}} \geq r \quad(k=1,2, \cdots) .
\end{aligned}
$$

Lemma 2.3. Let $A_{1} \in \mathcal{B}_{1}(\Omega)$ and let $n$ be a natural number. Given $\epsilon>0$, there exist $A_{k} \in \mathcal{B}_{1}(\Omega)(k=2,3, \cdots, n)$ and $K_{k}$ compact with $\left\|K_{k}\right\|<\frac{\epsilon}{2^{k}}(k=1,2, \cdots$, $n-1)$ such that $A_{k}=A_{k-1}+K_{k-1}(k=2,3, \cdots, n)$, $\operatorname{ker} \tau_{A_{i} A_{j}}=\{0\} \quad(i<j)$ and $A_{k}(k=1,2, \cdots, n)$ admits an upper triangular representation with respect to an $O N B\left\{e_{n}\right\}_{n=1}^{\infty}$ of $\mathcal{H}$.

Proof. Let $\lambda_{0}$ be in $\Omega$. From Lemma 2.2, $A_{1}$ admits an upper triangular matrix of the form

$$
A_{1}=\left(\begin{array}{ccccc}
\lambda_{0} & a_{12}^{1} & a_{13}^{1} & \ldots \\
& \lambda_{0} & a_{23}^{1} & \ldots & e_{1} \\
& & \lambda_{0} & \ldots \\
0 & & & \ldots
\end{array}\right) e_{2}
$$

with respect to an ONB $\left\{e_{n}\right\}_{n=1}^{\infty}$ of $\mathcal{H}$ and $\left|a_{k, k+1}^{1}\right|>r>0$ for some $r>0$. Fix a natural number $N$ so that $\left\|a_{k} A_{1}\right\|<\frac{\epsilon}{2}(k>N)$, where $\left(\frac{k+1}{k}\right)^{1 / 2}=1+a_{k}$. 
Set

$$
A_{2}=\left(\begin{array}{cccc|c}
\lambda_{0} & a_{12}^{2} & a_{13}^{2} & \ldots \\
& \lambda_{0} & a_{23}^{2} & \ldots & e_{1} \\
& & \lambda_{0} & \ldots \\
0 & & & \ldots
\end{array}\right) e_{2}
$$

with respect to the $\mathrm{ONB}\left\{e_{n}\right\}_{n=1}^{\infty}$, where

$$
\begin{aligned}
& a_{k, k+1}^{2}= \begin{cases}a_{k, k+1}^{1}, & k \leq N \\
\left(\frac{k+1}{k}\right)^{1 / 2} a_{k, k+1}^{1}, & k>N,\end{cases} \\
& a_{i j}^{2}=a_{i j}^{1}, \quad j \neq i+1 .
\end{aligned}
$$

Thus $A_{2}=A_{1}+K_{1}$ and $K_{1}$ is compact with $\left\|K_{1}\right\|<\frac{\epsilon}{2}$. Since $a_{k, k+1}^{2} \neq 0(k=$ $1,2, \cdots), \operatorname{dim} \operatorname{ker}\left(A_{2}-\lambda_{0}\right)^{*}=0$ and $\bigvee\left\{\operatorname{ker}\left(A_{2}-\lambda_{0}\right)^{k}: k=1,2, \cdots\right\}=\mathcal{H}$ Thus $A_{2} \in \mathcal{B}_{1}(\Omega)$. If $X \in \operatorname{ker} \tau_{A_{1} A_{2}}$, then $\left(A_{1}-\lambda_{0}\right) X=X\left(A_{2}-\lambda_{0}\right)$. From $\left(A_{1}-\lambda_{0}\right) X e_{1}=X\left(A_{2}-\lambda_{0}\right) e_{1}=0$ and $\operatorname{ker}\left(A_{1}-\lambda_{0}\right)=\bigvee\left\{e_{1}\right\}, X e_{1}=x_{11} e_{1}$ for some number $x_{11}$. Since $\left(A_{1}-\lambda_{0}\right)^{2} X e_{2}=0, X e_{2} \in \bigvee\left\{e_{1}, e_{2}\right\}$, i.e. $X e_{2}=x_{12} e_{1}+x_{22} e_{2}$. In general, we can prove that $X e_{k}=\sum_{i=1}^{k} x_{i k} e_{i}$, i.e. $X$ admits an upper triangular matrix representation with respect to the ONB $\left\{e_{n}\right\}_{n=1}^{\infty}$. Computations show that

$$
x_{k k}=\frac{\prod_{i=1}^{k-1} a_{i, i+1}^{2}}{\prod_{i=1}^{k-1} a_{i, i+1}^{1}} x_{11}= \begin{cases}\left(\frac{k}{N+1}\right)^{1 / 2} x_{11} & (k>N), \\ x_{11} & (k \leq N) .\end{cases}
$$

Since $\left|x_{k, k}\right| \leq\|X\|, x_{k, k}=0(k=1,2, \cdots)$.

Similarly,

$$
x_{k, k+1}=\frac{a_{k, k+1}^{2} \prod_{i=2}^{k-1} a_{i, i+1}^{2}}{a_{12}^{1} \prod_{i=2}^{k-1} a_{i, i+1}^{1}} x_{12}= \begin{cases}\frac{a_{k, k+1}^{2}}{a_{12}^{1}}\left(\frac{k}{N+1}\right)^{1 / 2} x_{12} & (k>N), \\ \frac{a_{k, k+1}^{2}}{a_{12}^{1}} x_{12} & (k \leq N) .\end{cases}
$$

Since $\left|a_{k, k+1}^{2}\right|>r>0$ and $\left|x_{k, k+1}\right| \leq\|X\|, x_{k, k+1}=0(k=1,2, \cdots)$. The same argument shows that $x_{i j}=0(j-i \geq 2)$, i.e. $X=0$ and $\operatorname{ker} \tau_{A_{1} A_{2}}=\{0\}$.

Define $A_{3}, \cdots, A_{n}$ inductively such that $A_{k}=A_{k-1}+K_{k-1}$ where $K_{k}$ is compact with $\left\|K_{k}\right\|<\frac{\epsilon}{2^{k}}$. The similar arguments indicate that $\left\{A_{k}\right\}_{k=1}^{n},\left\{K_{k}\right\}_{k=1}^{n-1}$ satisfy the requirements of the lemma.

Lemma 2.4. Let $\left\{A_{k}\right\}_{k=1}^{n}$ be given in Lemma 2.3 and let $\epsilon>0$; then there exists $Q_{k, k-1}$ compact with $\left\|Q_{k, k-1}\right\|<\epsilon$ such that $Q_{k, k-1} \notin \operatorname{ran} \tau_{A_{k} A_{k-1}}(k=$ $2,3, \cdots, n)$.

Proof. Set

$$
Q_{21}=\left(\begin{array}{ccccc}
0 & a_{1} & 0 & 0 & \ldots \\
0 & 0 & a_{2} & 0 & \ldots \\
0 & 0 & 0 & a_{3} & \ldots \\
& & & & \ddots
\end{array}\right) \begin{gathered}
e_{1} \\
e_{2} \\
e_{3} \\
\vdots
\end{gathered}
$$

where $a_{i}=\frac{\epsilon}{2 \sqrt{i}\left\|A_{2}\right\|^{2}} a_{i, i+1}^{2}(i=1,2, \cdots)$; then $Q_{21}$ is compact with $\left\|Q_{21}\right\|<\epsilon$. If $X \in \mathcal{L}(\mathcal{H})$ satisfies $A_{2} X-X A_{1}=Q_{21}$, then

$$
\left(A_{2}-\lambda_{0}\right) X e_{1}=Q_{21} e_{1}+X\left(A_{1}-\lambda_{0}\right) e_{1}=0
$$


Thus $X e_{1} \in \operatorname{ker}\left(A_{2}-\lambda_{0}\right)$, i.e. $X e_{1}=x_{11} e_{1}$ for some number $x_{11}$. Again, $\left(A_{2}-\lambda_{0}\right) X e_{2}=a_{1} e_{1}+X\left(A_{1}-\lambda_{0}\right) e_{2}$ and $X e_{2} \in \operatorname{ker}\left(A_{2}-\lambda_{0}\right)^{2}$. i.e. $X e_{2}=$ $x_{12} e_{1}+x_{22} e_{2}$. In general, $X e_{k}=x_{1 k} e_{1}+\cdots+x_{k k} e_{k}(k=1,2, \cdots)$ and $X$ has an upper triangular form with respect to $\left\{e_{n}\right\}_{n=1}^{\infty}$.

Computations indicate that

$$
\begin{aligned}
& x_{22}=\frac{a_{12}^{1}}{a_{12}^{2}} x_{11}+\frac{a_{1}}{a_{12}^{2}}, \\
& x_{33}=\frac{a_{12}^{1} a_{23}^{1}}{a_{12}^{2} a_{23}^{2}} x_{11}+\frac{a_{1}}{a_{12}^{2}} \cdot \frac{a_{23}^{1}}{a_{23}^{2}}+\frac{a_{2}}{a_{23}^{2}}, \\
& \ldots \ldots \ldots \cdots \cdots \\
& x_{k+1, k+1}= \prod_{i=1}^{k} \frac{a_{i, i+1}^{1}}{a_{i, i+1}^{2}} x_{11}+\frac{a_{1}}{a_{12}^{2}} \prod_{i=2}^{k} \frac{a_{i, i+1}^{1}}{a_{i, i+1}^{2}}+\frac{a_{2}}{a_{23}^{2}} \prod_{i=3}^{k} \frac{a_{i, i+1}^{1}}{a_{i, i+1}^{2}}+\cdots \\
& \quad+\frac{a_{k-1}}{a_{k-1, k}^{2}} \frac{a_{k, k+1}^{1}}{a_{k, k+1}^{2}}+\frac{a_{k}}{a_{k, k+1}^{2}} .
\end{aligned}
$$

From the proof of Lemma 2.3,

$$
a_{i, i+1}^{2}= \begin{cases}a_{i, i+1}^{1}, & i \leq N \\ \left(\frac{i+1}{i}\right)^{1 / 2} a_{i, i+1}^{1}, & i>N\end{cases}
$$

Thus, if $k>N$ we have

$$
\prod_{j=i}^{k} \frac{a_{j, j+1}^{1}}{a_{j, j+1}^{2}}= \begin{cases}\prod_{j=N+1}^{k}\left(\frac{j}{j+1}\right)^{1 / 2}=\left(\frac{N+1}{k+1}\right)^{1 / 2}, & i \leq N \\ \prod_{j=i}^{k}\left(\frac{j}{j+1}\right)^{1 / 2}=\left(\frac{i}{k+1}\right)^{1 / 2}, & i>N\end{cases}
$$

and

$$
\frac{a_{i}}{a_{i, i+1}^{2}} \prod_{j=i}^{k} \frac{a_{j, j+1}^{1}}{a_{j, j+1}^{2}} \geq \frac{\epsilon}{2\left\|A_{2}\right\|(k+1)^{1 / 2}} \quad(i=2, \cdots, k) .
$$

Thus $\left|x_{k+1, k+1}\right| \geq \frac{k \epsilon}{2\left\|A_{2}\right\|(k+1)^{1 / 2}} \rightarrow \infty \quad(k \rightarrow \infty)$. The contradiction indicates that $Q_{21} \notin \operatorname{ran} \tau_{A_{2} A_{1}}$. By the same arguments we can prove the conclusion for $k=3, \cdots, n$.

Lemma 2.5. Let $A_{1} \in \mathcal{B}_{1}(\Omega)$ and let $n$ be a natural number, $\epsilon>0$; then there exist $\bar{A}(\Omega) \in(S I), K, Q(\Omega)$ compact with $\|K\|<\epsilon$ and $\|Q(\Omega)\|<\epsilon$ such that

$$
\bar{A}(\Omega)=A_{1}^{(n-1)}+K \in \mathcal{B}_{n-1}(\Omega), Q(\Omega) \notin \operatorname{ran} \tau_{\bar{A}(\Omega), A_{1}}
$$

and $\operatorname{ker} \tau_{A_{1}, \bar{A}(\Omega)}=\{0\}$.

Proof. Assume that $A_{2}, \cdots, A_{n}$ are given in Lemma 2.3 such that

$$
A_{k}=A_{k-1}+K_{k-1}=A_{1}+\sum_{i=1}^{k-1} K_{i}=A_{1}+K_{k}^{\prime} .
$$

Thus $K_{k}^{\prime}$ is compact with $\left\|K_{k}^{\prime}\right\|<\epsilon$. Assume that $Q_{2,1}, \cdots, Q_{n, n-1}$ are given in Lemma 2.4 such that $Q_{k, k-1}$ is compact with $\left\|Q_{k, k-1}\right\|<\epsilon$ and $Q_{k, k-1} \notin$ $\operatorname{ran} \tau_{A_{k} A_{k-1}}(k=2,3, \cdots, n)$. 
Set

$$
\bar{A}(\Omega)=\left(\begin{array}{ccccc}
A_{n} & Q_{n, n-1} & & & \\
& A_{n-1} & Q_{n-1, n-2} & & \\
& & A_{n-2} & \ddots & \\
& & & \ddots & Q_{3,2} \\
0 & & & & A_{2}
\end{array}\right)
$$

on $\mathcal{H}^{(n-1)}$. Then $\bar{A}(\Omega)=A_{1}^{(n-1)}+K$, where

$$
K=\left(\begin{array}{ccccc}
K_{n}^{\prime} & Q_{n, n-1} & & & \\
& K_{n-1}^{\prime} & Q_{n-1, n-2} & & \\
& & K_{n-2}^{\prime} & \ddots & \\
& & & \ddots & Q_{3,2} \\
0 & & & & K_{2}^{\prime}
\end{array}\right)
$$

Thus it is obvious that $K$ is compact with $\|K\|<\epsilon$.

Suppose that $P \in \mathcal{A}^{\prime}(\bar{A}(\Omega))$ is an idempotent and

$$
P=\left(\begin{array}{ccc}
P_{n n} & \ldots & P_{n 2} \\
& \ldots & \\
P_{2 n} & \ldots & P_{22}
\end{array}\right) \begin{gathered}
\mathcal{H} \\
\vdots
\end{gathered}
$$

Then $P \bar{A}(\Omega)=\bar{A}(\Omega) P$ implies that

$$
P_{2 n} A_{n}=A_{2} P_{2 n} .
$$

Since $\operatorname{ker} \tau_{A_{2} A_{n}}=\{0\}, P_{2 n}=0$. By the same argument we can prove

$$
P_{i j}=0 \quad(i<j) .
$$

Thus $P_{i i} \in \mathcal{A}^{\prime}\left(A_{i}\right)(i=2, \cdots, n)$. Since each $\mathcal{B}_{1}(\Omega)$ operator is in (SI), $P_{i i}=\delta_{i} I$, where $\delta_{i}=0$ or 1 and $I$ is the identity of $\mathcal{H}$. Assume that $\delta_{n}=0$ (if $\delta_{n}=1$, consider $\left.I^{(n-1)}-P\right)$; then

$$
P_{n, n-1} A_{n-1}=A_{n} P_{n, n-1}+\delta_{n-1} Q_{n, n-1} .
$$

Since $Q_{n, n-1} \notin \operatorname{ran} \tau_{A_{n} A_{n-1}}, \delta_{n-1}=0$. Similarly $\delta_{i}=0(i=2,3, \cdots, n-2)$. Thus $P=0$ and $\bar{A}(\Omega) \in(\mathrm{SI})$. It is a routine exercise to check that $\bar{A}(\Omega) \in \mathcal{B}_{n-1}(\Omega)$.

Set

$$
Q(\Omega)=\left(\begin{array}{c}
0 \\
\vdots \\
0 \\
Q_{21}
\end{array}\right) \in \mathcal{L}\left(\mathcal{H}, \mathcal{H}^{(n-1)}\right)
$$

If $X \in \mathcal{L}\left(\mathcal{H}, \mathcal{H}^{(n-1)}\right)$ satisfies $\bar{A}(\Omega) X-X A_{1}=Q(\Omega)$ and assuming that

$$
X=\left(\begin{array}{c}
X_{1} \\
\vdots \\
X_{n-1}
\end{array}\right),
$$

then $A_{2} X_{n-1}-X_{n-1} A_{1}=Q_{21}$, i.e. $Q_{21} \in \operatorname{ran} \tau_{A_{2} A_{1}}$. Thus $Q(\Omega) \notin \operatorname{ran} \tau_{\bar{A}(\Omega) A_{1}}$. 
If $Y \in \mathcal{L}\left(\mathcal{H}^{(n-1)}, \mathcal{H}\right)$ satisfies $A_{1} Y=Y \bar{A}(\Omega)$ and assume that

$$
Y=\left(Y_{1}, Y_{2}, \cdots, Y_{n-1}\right) .
$$

Since $\operatorname{ker} \tau_{A_{i} A_{j}}=\{0\}(1 \leq i<j \leq n-1), Y_{i}=0(i=1,2, \cdots, n-1)$, i.e. $\operatorname{ker} \tau_{A_{1} \bar{A}(\Omega)}=\{0\}$.

Lemma 2.6. Given $A, B \in \mathcal{L}(\mathcal{H})$. Let $\Gamma=\left\{a_{n}\right\}_{n=1}^{\infty} \subset \sigma_{p}(A)$ and $\beta \in \sigma_{p}(B)$ satisfy

(i) $\beta \notin \sigma_{p}(A)$ but $\beta \in \bar{\Gamma}$;

(ii) $\operatorname{dim} \operatorname{ker}\left(A-a_{n}\right)<\infty(n=1,2, \cdots), \operatorname{dim} \operatorname{ker}(B-\beta)<\infty$;

(iii) $\bigvee\left\{\operatorname{ker}\left(A-a_{n}\right): n=1,2, \cdots\right\}=\bigvee\left\{\operatorname{ker}(B-\beta)^{n}: n=1,2, \cdots\right\}=\mathcal{H}$.

Then for each $\epsilon>0$, there exists $K$ compact with $\|K\|<\epsilon$ such that $K \notin \operatorname{ran} \tau_{A B}$.

Proof. From (ii), (iii), there is an ONB $\left\{e_{n}\right\}_{n=1}^{\infty}$ of $\mathcal{H}$ such that

$$
\begin{aligned}
& A=\left(\begin{array}{cccc}
a_{1} & & & \\
& a_{2} & & * \\
& & \ddots & \\
0 & & & e_{1} \\
e_{2} \\
\vdots \\
\vdots
\end{array}\right. \\
& U B U^{*}=B^{\prime}=\left(\begin{array}{llll}
\beta & & & \\
& \beta & & * \\
& & \ddots & \\
0 & & &
\end{array}\right) \begin{array}{c}
e_{1} \\
e_{2} \\
\vdots \\
\vdots
\end{array}
\end{aligned}
$$

where $U$ is an unitary. Assume that subsequence $\left\{a_{n_{k}}\right\}_{k=1}^{\infty}$ of $\left\{a_{n}\right\}_{n=1}^{\infty}$ satisfies

$$
\left|a_{n_{k}}-\beta\right|<\frac{1}{2^{k}} \quad(k=1,2, \cdots) .
$$

Let $K^{\prime} \in \mathcal{L}(\mathcal{H})$ be defined by $K^{\prime} e_{n}=\lambda_{n} e_{n}$, where

$$
\lambda_{n}= \begin{cases}\frac{\epsilon}{2 k}, & \text { if } n=n_{k} \text { for some } k \\ 0, & \text { otherwise. }\end{cases}
$$

Then $K^{\prime}$ is compact and $\left\|K^{\prime}\right\|<\epsilon$. If $X \in \mathcal{L}(\mathcal{H})$ satisfies $A X-X B^{\prime}=K^{\prime}$, then $(A-\beta) X e_{1}=X\left(B^{\prime}-\beta\right) e_{1}+K^{\prime} e_{1} \in \bigvee\left\{e_{1}\right\}$. Since $A-\beta$ is injective and upper triangular, $X e_{1}=x_{11} e_{1}$ for some number $x_{11}$. Similarly $(A-\beta) X e_{2}=$ $X\left(B^{\prime}-\beta\right) e_{2}+K^{\prime} e_{2} \in \bigvee\left\{e_{1}, e_{2}\right\}$, and thus $X e_{2}=x_{12} e_{1}+x_{22} e_{2}$. In general, $X e_{n}=x_{1 n} e_{1}+\cdots+x_{n n} e_{n}$ and $X$ admits an upper triangular representation with respect to the ONB $\left\{e_{n}\right\}_{n=1}^{\infty}$. Computation shows that $x_{n n}=\frac{\lambda_{n}}{a_{n}-\beta}(n=1,2, \cdots)$. Thus $\left|x_{n_{k} n_{k}}\right| \rightarrow \infty(k \rightarrow \infty)$. Therefore $K=K^{\prime} U \notin \operatorname{ran} \tau_{A B}$.

Lemma 2.7. Let $A, K \in \mathcal{L}(\mathcal{H})$ be given by $A e_{1}=0, A e_{n+1}=\frac{1}{n} e_{n}$ and $K e_{n}=$ $\frac{\epsilon}{2 \sqrt{n}} e_{n}(n=1,2, \cdots)$, where $\left\{e_{n}\right\}_{n=1}^{\infty}$ is an $O N B$ of $\mathcal{H}$. Then $K$ is compact with $\|K\|<\epsilon$ and $K \notin \operatorname{ran} \tau_{A A^{*}}$. 
Proof. If $A X-X A^{*}=K$ for some $X \in \mathcal{L}(\mathcal{H})$ and assuming that

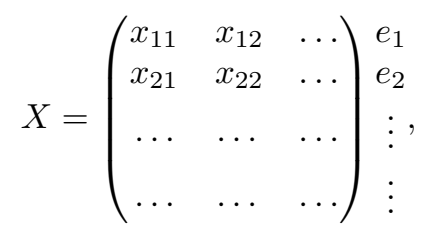

then $\frac{1}{n}\left(x_{n+1, n}-x_{n, n+1}\right)=\frac{\epsilon}{\sqrt{n}}$ and $\left|x_{n+1, n}-x_{n, n+1}\right|=\sqrt{n} \epsilon \rightarrow \infty(n \rightarrow \infty)$. Thus $K \notin \operatorname{ran} \tau_{A A^{*}}$.

Lemma 2.8. Given a domain $\Omega$, there exists an essentially normal and co-subnormal operator $N$ such that for each $\epsilon>0$, there is $K$ compact with $\|K\|<\epsilon$ satisfying

(i) $A(\Omega)=N+K \in \mathcal{B}_{1}(\Omega)$;

(ii) $\sigma(A(\Omega))=\bar{\Omega}$ and $\sigma_{e}(A)=\partial \Omega$;

(iii) $\sigma_{p}(A(\Omega)) \cap \partial \bar{\Omega}=\varnothing$.

Proof. Let $N_{+}\left(\Omega_{1}^{*}\right)$ be the Bergman operator on $\mathcal{H}_{0}=L_{a}^{2}\left(\Omega_{1}^{*}\right)$ and $S_{0}=N_{+}\left(\Omega_{1}^{*}\right)^{*}$, where $\Omega_{1}=\bar{\Omega}^{0}$. Denote $\sigma=\Omega_{1}-\Omega$. Let $\left\{\mu_{n}\right\}_{n=1}^{l}(0 \leq l \leq \infty)$ be a dense subset of $\sigma$ and let $\left\{\mathcal{H}_{n}\right\}_{n=1}^{\infty}$ be a sequence of Hilbert spaces and $\left\{e_{k}^{n}\right\}_{k=1}^{\infty}$ be an ONB of Hilbert space $\mathcal{H}_{n}(n=0,1,2, \cdots)$. Define $A_{n} e_{k}^{n}=\frac{\epsilon}{(k !)^{n}} e_{k+1}^{n}=a_{k}^{n} e_{k+1}^{n} \quad(k=$ $1,2, \cdots)$ and $S_{n}=\mu_{n} I_{n}+A_{n}$, where $I_{n}$ is the identity on $\mathcal{H}_{n}(n=1,2, \cdots)$. From a result of M. Cowen and R. G. Douglas [6], there is an analytic function $f: \Omega_{1} \rightarrow \mathcal{H}_{0}$ such that $f(\lambda) \neq 0$ and

$$
\left(S_{0}-\lambda\right) f(\lambda) \equiv 0 \quad\left(\lambda \in \Omega_{1}\right) .
$$

Define

$$
A(\Omega)=\left(\begin{array}{cccc}
S_{0} & & & 0 \\
C_{1} & S_{1} & & \\
C_{2} & 0 & S_{2} & \\
\vdots & & & \ddots
\end{array}\right) \begin{gathered}
\mathcal{H}_{0} \\
\mathcal{H}_{1} \\
\mathcal{H}_{2} \\
\vdots
\end{gathered}
$$

with respect to $\mathcal{H}=\bigoplus_{i=0}^{l} \mathcal{H}_{i}$, where

$$
C_{n}=\frac{\epsilon}{2^{n}\left\|f\left(\mu_{n}\right)\right\|} e_{1}^{n} \otimes f\left(\mu_{n}\right)^{*} \quad(n=1,2, \cdots) .
$$

Then $A(\Omega)=N+K$, where

$$
N=S_{0} \oplus\left(\bigoplus_{n=1}^{l} \mu_{n} I_{n}\right)
$$

is essentially normal and co-subnormal and

$$
K=\left(\begin{array}{cccc}
0 & & & 0 \\
C_{1} & A_{1} & & \\
C_{2} & 0 & A_{2} & \\
\vdots & & & \ddots
\end{array}\right)
$$

is compact with $\|K\|<\epsilon$. 
Thus $\sigma(A(\Omega)) \supset \Omega_{1}$, ind $(\lambda-A(\Omega))=1\left(\lambda \in \Omega_{1}\right)$ and $\sigma_{e}(A(\Omega))=\sigma_{e}(N)=\partial \Omega$. Since $\sigma_{p}\left(S_{n}^{*}\right) \cap \Omega=\varnothing(n=0,1,2, \cdots), \operatorname{ker}(\lambda-A(\Omega))^{*}=0\left(\lambda \in \Omega_{1}\right)$. Set

$$
\begin{array}{r}
x(\lambda)=f(\lambda) \oplus\left[-\frac{\epsilon\left(f(\lambda), f\left(\mu_{1}\right)\right)}{2 \| f\left(\mu_{1}\right) \mid} \sum_{k=1}^{\infty} \frac{(-1)^{k+1} a_{1}^{1} \cdots a_{k-1}^{1}}{\left(\mu_{1}-\lambda\right)^{k}} e_{k}^{1}\right] \oplus \cdots \\
\oplus\left[-\frac{\epsilon\left(f(\lambda), f\left(\mu_{n}\right)\right)}{2^{n}\left\|f\left(\mu_{n}\right)\right\|} \sum_{k=1}^{\infty} \frac{(-1)^{k+1} a_{1}^{n} \cdots a_{k-1}^{n}}{\left(\mu_{n}-\lambda\right)^{k}} e_{k}^{n}\right] \oplus \cdots .
\end{array}
$$

Then computation shows that $x(\lambda) \in \operatorname{ker}(A(\Omega)-\lambda)\left(\lambda \in \Omega_{1}\right)$.

Set $\bigvee\{x(\lambda): \lambda \in \Omega\}=\mathcal{H}^{\prime}$ and assume that $\bigoplus_{n=0}^{l} y_{n} \in \mathcal{H}^{\prime \perp}$, where $y_{n} \in \mathcal{H}_{n}$ and $y_{n}=\sum_{k=1}^{\infty} \bar{b}_{k}^{n} e_{k}^{n}(n=0,1,2, \cdots)$; then

$$
\begin{gathered}
\left(f(\lambda), y_{0}\right)=\frac{\epsilon\left(f(\lambda), f\left(\mu_{1}\right)\right)}{2\left\|f\left(\mu_{1}\right)\right\|} \sum_{k=1}^{\infty} \frac{(-1)^{k+1} a^{1} \cdots a_{k-1}^{1} b_{k}^{1}}{\left(\mu_{1}-\lambda\right)^{k}}+\cdots \\
+\frac{\epsilon\left(f(\lambda), f\left(\mu_{n}\right)\right)}{2^{n}\left\|f\left(\mu_{n}\right)\right\|} \sum_{k=1}^{\infty} \frac{a_{1}^{n} \cdots a_{k-1}^{n} b_{k}^{n}}{\left(\mu_{n}-\lambda\right)^{k}}+\cdots .
\end{gathered}
$$

Since $\left(f(\lambda), y_{0}\right)$ is analytic in $\Omega_{1}$ (including $\left.\mu_{k}(k=1,2, \cdots)\right)$ and since $f\left(\mu_{k}\right) \neq 0$, $b_{k}^{n}=0(k=1,2, \cdots ; n=1,2, \cdots)$. Therefore $\bigvee\{x(\lambda): \lambda \in \Omega\}=\mathcal{H}$ and $A(\Omega) \in \mathcal{B}_{1}(\Omega)$.

If $\lambda \notin \bar{\Omega}$, then $\lambda \in \rho(N)$. Since $\lambda \notin \sigma_{p}\left(A(\Omega)^{*}\right), \lambda \in \rho(A(\Omega))$ and $\sigma(A(\Omega))=\bar{\Omega}$. Since $\sigma_{p}\left(S_{0}\right) \cap \partial \Omega_{1}=\varnothing$ and since $\partial \Omega_{1} \subset \rho\left(S_{n}\right)(n=1,2, \cdots), \sigma_{p}(A(\Omega)) \cap \partial \Omega_{1}=$ $\varnothing$.

Given a compact subset $\sigma$ of $C$, consider the Sobolev space

$$
W^{22}(\mathcal{D})=\left\{f \in L^{2}(\mathcal{D}, d A): \begin{array}{l}
\text { the distributional partial derivatives of first } \\
\text { and second order of } f \text { belong to } L^{2}(\mathcal{D}, d A)
\end{array}\right\},
$$

where $\mathcal{D}=(a, b)^{2} \supset \sigma$ and $d A$ denotes the planar Lebesgue measure. It is well known that $W^{22}(\mathcal{D})$ is a Hilbert space of continuous functions (by Sobolev's embedding theorem) under the norm

$$
\|f\|_{W^{22}(\mathcal{D})}=\left(\int_{\mathcal{D}} \sum_{|\alpha| \leq 2}\left|D^{\alpha} f\right|^{2} d A\right)^{1 / 2},
$$

$W^{22}(D)$ is a regular Banach algebra with identity under pointwise multiplication and an equivalent norm whose maximal ideal space can be naturally identified with $\overline{\mathcal{D}}$ via "point evaluations".

Consider the subalgebra $W_{0}^{22}(\mathcal{D})$ of $W^{22}(\mathcal{D})$

$$
W_{0}^{22}(\mathcal{D})=\left\{f \in W^{22}(\mathcal{D}),\left.f\right|_{\partial \mathcal{D}} \equiv 0\right\} .
$$

For each $f \in W^{22}(\mathcal{D})$, the operator $M_{f}^{0} \in \mathcal{L}\left(W_{0}^{22}(\mathcal{D})\right)$ is given by

$$
M_{f}^{0} g(\lambda)=f(\lambda) g(\lambda), g \in W_{0}^{22}(\mathcal{D}) .
$$

Set $m_{0}(\sigma)=\left\{f \in W_{0}^{22}(\mathcal{D}):\left.f\right|_{\sigma} \equiv 0\right\}$. Then it is clear that $m_{0}(\sigma)$ leaves invariant under $M_{\lambda}^{0}$, i.e.

$$
M_{\lambda}^{0}=\left(\begin{array}{cc}
\left.M_{\lambda}^{0}\right|_{m_{0}(\sigma)} & * \\
0 & M_{\lambda}^{0}(\sigma)
\end{array}\right)
$$


with respect to the decomposition $W_{0}^{22}(\mathcal{D})=m_{0}(\sigma) \oplus\left[W_{0}^{22}(\mathcal{D}) \ominus m_{0}(\sigma)\right]$, where $M_{\lambda}^{0}$ is the operator "multiplication by $\lambda$ " and $M_{\lambda}^{0}(\sigma)$ is the compression of $M_{\lambda}^{0}$ to $W_{0}^{22}(\mathcal{D}) \ominus m_{0}(\sigma)$.

Lemma 2.9 ([20, Propositions 3.1, 3.2]). $\quad$ (i) $\sigma\left(M_{\lambda}^{0}(\sigma)\right)=\sigma_{e}\left(M_{\lambda}^{0}(\sigma)\right)=\sigma$;

(ii) $\operatorname{nul}\left(\lambda-M_{\lambda}^{0}(\sigma)\right)=0$ and $\operatorname{nul}\left(\lambda-M_{\lambda}^{0}(\sigma)\right)^{*}=1(\lambda \in \sigma)$;

(iii) For each dense subset $\left\{\lambda_{i}\right\}_{i=1}^{\infty}$ of $\sigma, \bigvee_{i=1}^{\infty} \operatorname{ker}\left(\lambda_{i}-M_{\lambda}^{0}(\sigma)\right)^{*}=W_{0}^{22}(\mathcal{D}) \ominus m_{0}(\sigma)$;

(iv) $M_{\lambda}^{0}(\sigma) \simeq$ normal+compact;

(v) If $\sigma$ is connected, then $M_{\lambda}^{0}(\sigma) \in(S I)$.

Denote $W_{0}(\sigma)=\left\{M_{f}^{0}(\sigma): f \in W^{22}(\mathcal{D})\right\}$; then it is easy to see that $W_{0}(\sigma)$ is a strictly cyclic operator algebra, where $M_{f}^{0}(\sigma)$ is the compression of $M_{f}^{0}$, the multiplication by function $f$, to the subspace $W_{0}^{22}(\mathcal{D}) \ominus m_{0}(\sigma)$. It was proved in [20] that $\mathcal{A}^{\prime}\left(M_{\lambda}^{0}(\sigma)\right)=W_{0}(\sigma)$ and there is a vector $f_{\mu} \in \operatorname{ker}\left(M_{\lambda}^{0}(\sigma)-\mu\right)^{*}$ such that $\left(f, f_{\mu}\right)=f(\mu)$ for each $f \in W_{0}^{22}(\mathcal{D}) \ominus m_{0}(\sigma)$ and $\mu \in \sigma$. Thus, if $P$ is an idempotent and $P M_{\lambda}^{0}(\sigma)^{*}=M_{\lambda}^{0}(\sigma)^{*} P$, then $P f_{\mu} \in \operatorname{ker}\left(M_{\lambda}^{0}(\sigma)-\mu\right)^{*}$. Since $\operatorname{dim} \operatorname{ker}\left(M_{\lambda}^{0}(\sigma)-\mu\right)^{*}=1, P f_{\mu}=a(\mu) f_{\mu}$ for some number $a(\mu)$. Since $P^{2}=P, a(\mu)=0$ or $1(\mu \in \sigma)$.

Lemma 2.10. Let $\Omega$ be a bounded domain, and let $A$ be an operator on $\mathcal{H}$ in $\mathcal{B}_{1}(\Omega)$ such that $\sigma(A)=\bar{\Omega}$ and $\sigma_{p}(A) \cap \partial \bar{\Omega}=\varnothing$. Also let $\sigma$ be a compact subset of the plane such that the components $\left\{\sigma_{n}\right\}_{n \in \Lambda}$ of $\sigma$ each have more than one point. Let $\sigma^{\prime}$ denote the union of those components of $\sigma$ which intersect $\partial \bar{\Omega}$. Let $\mathcal{D}$ be an open square containing $\sigma^{*}$, and set $B=M_{\lambda}^{0}\left(\sigma^{*}\right)^{*}$ acting on $\mathcal{H}_{1}=W^{22}(\mathcal{D}) \ominus m_{0}\left(\sigma^{*}\right)$. Then given $\epsilon>0$, there is a compact operator $D \in \mathcal{L}\left(\mathcal{H}_{1}, \mathcal{H}\right)$ with $\|D\|<\epsilon$ such that $D \notin \operatorname{ran} \tau_{A B}$ and having the property: if there are an idempotent $P$ in $\mathcal{A}^{\prime}(B)$ and an operator $X \in \mathcal{L}\left(\mathcal{H}_{1}, \mathcal{H}\right)$ such that $A X P-X P B=D P$, then $\left.a(\lambda)\right|_{\sigma^{\prime *}}=0$ and $P f_{\mu}=a(\mu) f_{\mu}$ for $\mu \in \sigma^{*}$.

Similarly, there is a compact operator $D^{\prime} \in \mathcal{L}\left(\mathcal{H}, \mathcal{H}_{1}\right)$ with $\left\|D^{\prime}\right\|<\epsilon$ such that $D^{\prime} \notin \operatorname{ran} \tau_{B A}$ and having the property: if there are an idempotent $P$ in $\mathcal{A}^{\prime}(B)$ and an operator $X \in \mathcal{L}\left(\mathcal{H}, \mathcal{H}_{1}\right)$ such that $B P X-P X A=P D$, then $\left.a(\lambda)\right|_{\sigma^{\prime *}}=0$ and $P f_{\mu}=a(\mu) f_{\mu}$ for $\mu \in \sigma^{*}$.

Proof. Choose two sets $\left\{\lambda_{i}^{1}\right\}_{i=1}^{\infty}$ and $\left\{\mu_{i}^{1}\right\}_{i=1}^{\infty}$ of distinct complex numbers satisfying

(i) $\left\{\lambda_{i}^{1}\right\}_{i=1}^{\infty} \subset \Omega$ and $\left\{\mu_{i}^{1}\right\}_{i=1}^{\infty} \subset \sigma^{\prime}$;

(ii) $\operatorname{card}\left\{i, \mu_{i}^{1} \in \sigma_{n}\right\}=\infty$ for each $\sigma_{n}, \sigma_{n} \cap \partial \bar{\Omega} \neq \varnothing$;

(iii) $\left|\mu_{i}^{1}-\lambda_{i}^{1}\right|<\frac{1}{2^{i}}(i=1,2, \cdots)$.

Choose dense subsets $\left\{\lambda_{k}\right\}_{i=1}^{\infty}$ of $\Omega$ and $\left\{\mu_{k}\right\}_{i=1}^{\infty}$ of $\sigma$ such that $\lambda_{i}^{1}=\lambda_{2 i}, \mu_{i}^{1}=\mu_{2 i}$ $(i=1,2, \cdots)$ and $\lambda_{i} \neq \lambda_{j}, \mu_{i} \neq \mu_{j}(i \neq j)$. Since $\bigvee\left\{\operatorname{ker}\left(\lambda_{i}-A\right): i=1,2, \cdots\right\}=\mathcal{H}$ and $\bigvee\left\{\operatorname{ker}\left(\mu_{i}-B\right): i=1,2, \cdots\right\}=\mathcal{H}_{1}$, it is completely apparent that $A$ and $B$ have upper triangular matrices with respect to ONB $\left\{e_{n}\right\}_{n=1}^{\infty}$ of $\mathcal{H}$ and respectively, $\left\{f_{n}\right\}_{n=1}^{\infty}$ of $\mathcal{H}_{1}$ obtained by Gram-Schmidt orthnormalization of unit vectors in $\bigcup_{i=1}^{\infty}\left\{\operatorname{ker}\left(\lambda_{i}-A\right)\right\}$ and $\bigcup_{i=1}^{\infty}\left\{\operatorname{ker}\left(\mu_{i}-B\right)\right\}$ respectively

$$
\begin{gathered}
A=\left(\begin{array}{cccc}
\lambda_{1} & & & * \\
& \lambda_{2} & & \\
0 & & \ddots & e_{1} \\
e_{2}, \\
\vdots
\end{array}\right. \\
B=\left(\begin{array}{cccc}
\mu_{1} & & & * \\
& \mu_{2} & & f_{1} \\
f_{2} \\
0 & & \ddots & \vdots
\end{array}\right)
\end{gathered}
$$


and

$$
U B U^{*}=B^{\prime}=\left(\begin{array}{cccc}
\mu_{1} & & & * \\
& \mu_{2} & & \\
0 & & \ddots &
\end{array}\right) \begin{gathered}
e_{1} \\
e_{2} \\
\vdots
\end{gathered}
$$

where $U$ is an unitary, $U f_{i}=e_{i}(i=1,2, \cdots)$.

Define $D^{\prime} e_{k}=a_{k} e_{k}$ where

$$
a_{k}= \begin{cases}\frac{\epsilon}{2 i}, & \text { if } k=2 i, \\ 0, & \text { otherwise }\end{cases}
$$

then $D^{\prime}$ is compact with $\left\|D^{\prime}\right\|<\epsilon$.

If $A X-X B^{\prime}=D^{\prime}$ for some $X$, then calculations show that $X$ admits an upper triangular matrix with respect to $\left\{e_{n}\right\}_{n=1}^{\infty}$. Thus $\left|x_{2 i, 2 i}\right|=\frac{a_{2 i}}{\left|\lambda_{2 i}-\mu_{2 i}\right|} \geq \frac{2^{i-1} \epsilon}{i} \rightarrow \infty$ $(i \rightarrow \infty)$, where $x_{2 i, 2 i}=\left(X e_{2 i}, e_{2 i}\right)(i=1,2, \cdots)$.

Therefore $D^{\prime} \notin \operatorname{ran} \tau_{A B^{\prime}}$ and $D=D^{\prime} U \notin \operatorname{ran} \tau_{A B}$. Suppose that $P \in \mathcal{A}^{\prime}(B)$ is an idempotent and $P f_{\mu}=a(\mu) f_{\mu}\left(\mu \in \sigma^{*}\right)$. If the function $a(\mu)$ takes two values 1 and 0 on a $\sigma_{n}^{*}$, then there is a sequence $\left\{h_{k}\right\}_{k=0}^{\infty} \subset \sigma_{n}$ such that $h_{k} \rightarrow h_{0}(k \rightarrow \infty)$, $a\left(\bar{h}_{k}\right)=0(k \geq 1)$ and $a\left(\bar{h}_{0}\right)=1$ (if $a\left(\bar{h}_{k}\right)=1(k \geq 1)$ and $a\left(\bar{h}_{0}\right)=0$, consider the idempotent $I_{1}-P$, where $I_{1}$ is the identity on $\left.\mathcal{H}_{1}\right)$.

Set $\mathcal{M}=\bigvee\left\{f_{\bar{\mu}}: a(\bar{\mu})=1, \mu \in \sigma\right\}, \mathcal{N}=\bigvee\left\{f_{\bar{\mu}}: a(\bar{\mu})=0, \mu \in \sigma\right\}$; then $f_{\bar{h}_{k}} \in \mathcal{M}(k \geq 1)$ and $f_{\bar{h}_{0}} \in \mathcal{N}$. Since $\mathcal{M} \subset \operatorname{ran} P$ and $\mathcal{N} \subset \operatorname{ker} P, \mathcal{M} \cap \mathcal{N}=\{0\}$.

Since $f_{\bar{h}_{k}} \rightarrow f_{\bar{h}_{0}}$ weakly, $f_{\bar{h}_{0}} \in \mathcal{M} \cap \mathcal{N}$. This contradiction implies that $a(\mu)$ takes the same value ( 1 or 0$)$ on each $\sigma_{n}^{*}$. Similar arguments show that $a(\mu)$ is continuous on $\sigma_{n}^{*}$.

Assume that $P$ satisfies $A X P-X P B=D P$ for some $X \in \mathcal{L}\left(\mathcal{H}_{1}, \mathcal{H}\right)$. Note that $P$ has an upper triangular matrix representation

$$
P=\left(\begin{array}{lll}
p_{1} & & * \\
& p_{2} & \\
0 & & \ddots
\end{array}\right)
$$

with respect to the ONB $\left\{f_{i}\right\}_{i=1}^{\infty}$. Thus $X P$ admits an upper triangular matrix form

$$
X P=\left(\begin{array}{lll}
y_{1} & & * \\
& y_{2} & \\
0 & & \ddots
\end{array}\right)
$$

with respect to the ONB's $\left\{e_{i}\right\}_{i=1}^{\infty}$ and $\left\{f_{i}\right\}_{i=1}^{\infty}$.

Suppose that $\sigma_{n} \cap \partial \bar{\Omega} \neq 0$ and $a(\bar{\mu})=1\left(\mu \in \sigma_{n}\right)$. Also, suppose that $\mu_{i_{j}}^{1} \in \sigma_{n}$ $(j=1,2, \cdots)$. Thus $A X P-X P B=D P$ indicates that $\lambda_{2 i_{j}} y_{2 i_{j}}-y_{2 i_{j}} \mu_{2 i_{j}}=$ $a_{2 i_{j}} p_{2 i_{j}}$.

Since $a\left(\bar{\mu}_{2 i_{j}}\right)=1, p_{2 i_{j}}=1$. Thus $y_{2 i_{j}}=\frac{2^{i_{j}-1} \epsilon}{i_{j}} \rightarrow \infty(j \rightarrow \infty)$. This contradiction implies that $\left.a(\mu)\right|_{\sigma^{\prime *}}=0$. Same arguments work in the case that $P$ satisfies $B P X-P X A=P D$ for some $X \in \mathcal{L}\left(\mathcal{H}, \mathcal{H}_{1}\right)$. 


\section{The proof of the Main Theorem}

Let $T$ be an essentially normal operator with connected spectrum. The spectral picture of $T$ is determined by the sets

$$
\begin{aligned}
\rho_{F}^{0}(T) \cap \sigma(T) & :=\{\lambda \in \sigma(T): \operatorname{ind}(T-\lambda I)=0\}=\bigcup_{i \in \Lambda_{1}} \Omega_{1 i} \\
\rho_{F}^{+}(T) & :=\{\lambda \in \sigma(T): \operatorname{ind}(T-\lambda I)>0\}=\bigcup_{i \in \Lambda_{2}} \Omega_{2 i}, \\
\rho_{F}^{-}(T) & :=\{\lambda \in \sigma(T): \operatorname{ind}(T-\lambda I)<0\}=\bigcup_{i \in \Lambda_{3}} \Omega_{3 i}, \\
\sigma & =\sigma(T) \backslash \operatorname{int}\left[\rho_{F}(T)^{-}\right]
\end{aligned}
$$

where $\Omega_{1 i}, \Omega_{2 i}, \Omega_{3 i}$ are connected components.

Choose $\bigcup_{l \in \Lambda_{4}} \sigma_{l} \subset \sigma$, where $\sigma_{l}$ is a connected compact set consisting of more than one point, $\sigma_{l} \cap \sigma_{l^{\prime}}=\varnothing\left(l \neq l^{\prime}\right)$ and for each $l$, there exists at least one connected component $\Omega$ of $\rho_{F}(T) \cap \sigma(T)$ such that $\sigma_{l} \cap \partial \Omega \neq \varnothing$ and $\sigma=\overline{\bigcup_{l \in \Lambda_{4}} \sigma_{l}}$.

Construct operators $A\left(\Omega_{1 i}\right) \in \mathcal{B}_{1}\left(\Omega_{1 i}\right)$ and $A\left(\Omega_{1 i}^{*}\right) \in \mathcal{B}_{1}\left(\Omega_{1 i}^{*}\right)$ (Lemma 2.8) with respect to $\Omega_{1 i}$ and, respectively, $\Omega_{1 i}^{*}\left(i \in \Lambda_{1}\right)$ and let the norm of the compact operator $K$ (in Lemma 2.8) be so small that $\bigoplus_{i \in \Lambda_{1}} A\left(\Omega_{1 i}\right)$ and $\bigoplus_{i \in \Lambda_{1}} A\left(\Omega_{1 i}^{*}\right)$ are sums of an essentially normal and co-subnormal operator and a compact operator. Set $C\left(\Omega_{1 i}\right)=A\left(\Omega_{1 i}^{*}\right)^{*}\left(i \in \Lambda_{1}\right)$.

For each domain $\Omega_{2 j}$, according to Lemma 2.5 and Lemma 2.8 construct operator $A\left(\Omega_{2 j}\right) \in B_{1}\left(\Omega_{2 j}\right)$ and compact operator $Q\left(\Omega_{2 j}\right)$. Let

$$
n_{j}=\operatorname{ind}(T-\lambda) \text { for } \lambda \in \Omega_{2 j} \text {. }
$$

Using Lemma 2.8 construct operator

$$
\bar{A}\left(\Omega_{2 j}\right) \in B_{n_{j}-1}\left(\Omega_{2 j}\right)
$$

if $n_{j}>1$ such that $Q\left(\Omega_{2 j}\right) \notin \tau_{\bar{A}\left(\Omega_{2 j}\right) A\left(\Omega_{2 j}\right)}$ and $\operatorname{ker} \tau_{A\left(\Omega_{2 j}\right) \bar{A}\left(\Omega_{2 j}\right)}=\{0\}$ and the norm of $Q\left(\Omega_{2 j}\right)$ is so small that $\bigoplus Q\left(\Omega_{2 j}\right)$ is compact. Let the norm of the compact operator $K$ in Lemma 2.8 and Lemma 2.5 be so small that $\bigoplus_{j \in \Lambda_{2}} A\left(\Omega_{2 j}\right)$ and $\bigoplus \bar{A}\left(\Omega_{2 j}\right)$ are sums of an essentially normal co-subnormal operator and a compact operator.

For each domain $\Omega_{3 k}$, according to Lemma 2.8 construct operators $A\left(\Omega_{3 k}\right) \in$ $\mathcal{B}_{1}\left(\Omega_{3 k}\right)$ and $A\left(\Omega_{3 k}^{*}\right) \in \mathcal{B}_{1}\left(\Omega_{3 k}^{*}\right)$. Let $n_{k}=\operatorname{ind}(T-\lambda)$ for $\lambda \in \Omega_{3 k}$. Using Lemma 2.5 and Lemma 2.8, construct operators $\bar{A}\left(\Omega_{3 k}^{*}\right) \in \mathcal{B}_{\left|n_{k}\right|}\left(\Omega_{3 k}^{*}\right)$ and $Q\left(\Omega_{3 k}^{*}\right)$ compact if $\left|n_{k}\right|>1$ such that

$$
Q\left(\Omega_{3 k}^{*}\right) \notin \operatorname{ran} \tau_{\bar{A}\left(\Omega_{3 k}^{*}\right) A\left(\Omega_{3 k}^{*}\right)}, \quad \operatorname{ker} \tau_{A\left(\Omega_{3 k}^{*}\right) \bar{A}\left(\Omega_{3 k}^{*}\right)}=\{0\}
$$

and the norm of $Q\left(\Omega_{3 k}^{*}\right)$ is so small that $\bigoplus Q\left(\Omega_{3 k}^{*}\right)$ is compact. Let the norm of the compact operator $K$ in Lemma 2.5 and Lemma 2.8 is so small that $\bigoplus_{k \in \Lambda_{3}} A\left(\Omega_{3 k}\right)$, $\bigoplus_{k \in \Lambda_{3}} A\left(\Omega_{3 k}^{*}\right)$ and $\bigoplus \bar{A}\left(\Omega_{3 k}^{*}\right)$ are sums of an essentially normal co-subnormal operator and a compact operator.

Set $C\left(\Omega_{3 k}\right)=A\left(\Omega_{3 k}^{*}\right)^{*}$ and $\bar{C}\left(\Omega_{3 k}\right)=\bar{A}\left(\Omega_{3 k}^{*}\right)^{*}$.

Construct $B=\left[M_{\lambda}^{0}\left(\sigma^{*}\right)\right]^{*}$ on Hilbert space $W_{0}^{22}(\mathcal{D}) \ominus m_{0}\left(\sigma^{*}\right)$ where $\mathcal{D}=(a, b)^{2} \supset$ $\sigma^{*}$. 


\section{The first step:}

Rearrange

$$
\left\{A\left(\Omega_{2 j}\right): j \in \Lambda_{2}\right\}=\left\{A_{k}\right\}_{k=1}^{l_{1}} \quad\left(0 \leq l_{1} \leq \infty\right)
$$

and

$$
\left\{A\left(\Omega_{1 j}\right): j \in \Lambda_{1}, A\left(\Omega_{3 k}\right) ; k \in \Lambda_{3}\right\}=\left\{A_{k}^{a}\right\}_{k=1}^{l_{2}} \quad\left(0 \leq l_{2} \leq \infty\right) .
$$

Thus $\bigoplus_{k=1}^{l_{1}} A_{k}$ and $\bigoplus_{k=1}^{l_{2}} A_{k}^{a}$ are essentially normal and co-subnormal + compact type. Therefore,

$$
\sigma\left[\bigoplus_{k=1}^{l_{1}} A_{k}\right]=\left[\bigcup_{k=1}^{l_{1}} \sigma\left(A_{k}\right)\right]^{-}, \quad \sigma\left[\bigoplus_{k=1}^{l_{2}} A_{k}^{a}\right]=\left[\bigcup_{k=1}^{l_{2}} \sigma\left(A_{k}^{a}\right)\right]^{-} .
$$

Define

$$
G=\left(\begin{array}{ccc}
\oplus A_{k} & D_{1} & 0 \\
0 & B & D_{2} \\
0 & 0 & \oplus A_{k}^{a}
\end{array}\right) \quad \text { on } \mathcal{H}_{1} \oplus \mathcal{H}_{2} \oplus \mathcal{H}_{3},
$$

where $\mathcal{H}_{1}=\bigoplus_{k=1}^{l_{1}} \mathcal{H}_{k}^{1}, \mathcal{H}_{2}=W_{0}^{22}(D) \ominus m_{0}\left(\sigma^{*}\right), \mathcal{H}_{3}=\bigoplus_{k=1}^{l_{2}} \mathcal{H}_{k}^{3}$, and for each $k, A_{k} \in \mathcal{L}\left(\mathcal{H}_{k}^{1}\right), A_{k}^{a} \in \mathcal{L}\left(\mathcal{H}_{k}^{3}\right)$. Assume that

$$
D_{1}=\left(\begin{array}{c}
D_{1}^{1} \\
D_{2}^{1} \\
\vdots
\end{array}\right) \quad \text { and } \quad D_{2}=\left(D_{1}^{2}, D_{2}^{2}, \cdots\right) \text {. }
$$

Choose $D_{k}^{1} \notin \operatorname{ran} \tau_{A_{k} B}$ and $D_{k}^{2} \notin \operatorname{ran} \tau_{B A_{k}^{a}}, D_{k}^{1}, D_{k}^{2}$ are compact and the norms of them are so small that $D_{1}, D_{2}$ are compact (Lemma 2.10).

Suppose that $P \in \mathcal{A}^{\prime}(G)$ is an idempotent and

$$
P=\left(\begin{array}{lll}
P_{11} & P_{12} & P_{13} \\
P_{21} & P_{22} & P_{23} \\
P_{31} & P_{32} & P_{33}
\end{array}\right) \mathcal{H}_{1}
$$

Then $P_{31}=0, P_{21}=0$ and $P_{32}=0$ by Lemma 2.1. Since $A_{k} \in(\mathrm{SI})$ and $A_{k}^{a} \in(\mathrm{SI})(k=1,2, \cdots)$, from Lemma $2.1, P_{11}=\bigoplus_{i=1}^{l_{1}} \delta_{i}^{1} I_{i}^{1}$ and $P_{33}=\bigoplus_{i=1}^{l_{2}} \delta_{i}^{3} I_{i}^{3}$, where $\delta_{i}^{1}\left(\delta_{i}^{3}\right)$ is 0 or 1 , and $I_{i}^{1}\left(I_{i}^{3}\right)$ is the identity operator on $\mathcal{H}_{i}^{1}\left(\mathcal{H}_{i}^{3}\right), i=1,2, \cdots$. Since $P_{22} \in \mathcal{A}^{\prime}(B)$ is an idempotent, $P_{22} f_{\mu}=a(\bar{\mu}) f_{\mu}(\mu \in \sigma)$ and $a(\mu)$ takes constant (1 or 0) on each $\sigma_{l}$ (Lemma 2.10). Set $\Delta_{1}=\left\{\sigma_{l}:\left.a(\mu)\right|_{\sigma_{l}^{*}} \equiv 0\right\}, \Delta_{2}=$ $\left\{\sigma_{l}:\left.a(\mu)\right|_{\sigma_{l}^{*}} \equiv 1\right\}$ and denote $\Gamma_{1}=\bigcup_{\Delta_{1}} \sigma_{l}, \Gamma_{2}=\bigcup_{\Delta_{2}} \sigma_{l}$. Thus $\Gamma_{1} \cup \Gamma_{2}=\sigma$ and $\Gamma_{1} \cap \Gamma_{2}=\varnothing$. Rearrange $\left\{\Omega_{1 i}\left(i \in \Lambda_{1}\right), \Omega_{2 i}\left(i \in \Lambda_{2}\right), \Omega_{3 i}\left(i \in \Lambda_{3}\right)\right\}=\left\{\Omega_{k}\right\}_{k=1}^{l}$, set $\Delta_{3}=\left\{\bar{\Omega}: a(\bar{\mu}) \equiv 0, \mu \in \partial \bar{\Omega}_{k}\right\}, \Delta_{4}=\left\{\bar{\Omega}_{k}: a(\bar{\mu}) \equiv 1, \mu \in \partial \bar{\Omega}_{k}\right\}$. Denote $\Sigma_{1}=\Gamma_{1} \cup\left[\bigcup_{\Delta_{3}} \bar{\Omega}_{k}\right]$ and $\Sigma_{2}=\Gamma_{2} \cup\left[\bigcup_{\Delta_{4}} \bar{\Omega}_{k}\right]$. Thus $\sigma(T)=\bar{\Sigma}_{1} \cup \bar{\Sigma}_{2}$. If $\mu_{0} \in$ $\bar{\Sigma}_{1} \cup \bar{\Sigma}_{2}, \mu_{0} \in \sigma$, since $\mu_{0}$ is not in any $\left(\bar{\Omega}_{k}\right)^{0}$. Suppose that $\mu_{0} \in \Gamma_{1}$. Since $\mu_{0} \in \bar{\Sigma}_{2}$, it is always possible to find a sequence $\left\{\mu_{k}\right\}_{k=1}^{\infty} \subset \Gamma_{2}$ such that $\mu_{k} \rightarrow \mu_{0}$. Since $a(\bar{\mu})$ is continuous, $a\left(\bar{\mu}_{0}\right)=1$. Similarly, if $\mu_{0} \in \Gamma_{2}$, we get $a\left(\bar{\mu}_{0}\right)=0$. The contradiction implies that $\bar{\Sigma}_{1} \cap \bar{\Sigma}_{2}=\varnothing$. Since $\sigma(T)=\sigma(G)$ is connected, one of $\bar{\Sigma}_{1}$ and $\bar{\Sigma}_{2}$ must be empty. Thus one of $\Gamma_{1}$ and $\Gamma_{2}$ must be empty and $a(\bar{\mu}) \equiv 0$ or $1(\mu \in \sigma)$, i.e. $P_{22}=\delta_{2} I_{2}, \delta_{2}=0$ or 1 , where $I_{2}$ is the identity operator on $\mathcal{H}_{2}$. 
Assume that

$$
P_{12}=\left(\begin{array}{c}
P_{1}^{1} \\
P_{2}^{1} \\
\vdots
\end{array}\right), \quad P_{23}=\left(P_{1}^{2}, P_{2}^{2}, \cdots\right)
$$

Then

$$
\left(\bigoplus \delta_{k}^{1} I_{k}^{1}\right) D_{1}+P_{12} B=\left(\bigoplus A_{k}\right) P_{12}+D_{1} P_{22}
$$

and

$$
P_{22} D_{2}+P_{23}\left(\bigoplus A_{k}^{a}\right)=B P_{23}+D_{2}\left(\bigoplus \delta_{k}^{3} I_{k}^{3}\right)
$$

imply

$$
A_{k} P_{k}^{1}-P_{k}^{1} B=D_{k}^{1}\left(\delta_{k}^{1}-\delta_{2}\right) I_{2}\left(k=1,2, \cdots, l_{1}\right)
$$

and

$$
B P_{k}^{2}-P_{k}^{2} A_{k}^{2}=\left(\delta_{2}-\delta_{k}^{3}\right) I_{2} D_{k}^{2}\left(k=1,2, \cdots, l_{2}\right) .
$$

Using Lemma 2.10 with respect to the idempotents $\left(\delta_{k}^{1}-\delta_{2}\right) I_{2}\left(k=1,2, \cdots, l_{1}\right)$ and $\left(\delta_{2}-\delta_{k}^{3}\right) I_{2}\left(k=1,2, \cdots, l_{2}\right)$, we know that $\delta_{k}^{1}=\delta_{k}^{2}=\delta_{2}$ for all $k$. i.e. $P=0$ or $I$, where $I$ is the identity operator on $\mathcal{H}_{1} \oplus \mathcal{H}_{2} \oplus \mathcal{H}_{3}$. Thus $G \in(\mathrm{SI})$.

The second step: Recovery of the positive indices.

Rearrange and reexpress $\left\{\bar{A}\left(\Omega_{2 j}\right)\right\}=\left\{\bar{A}_{k}\right\}$. Then $\sigma\left(\bigoplus \bar{A}_{k}\right)=\left[\bigcup \sigma\left(\bar{A}_{k}\right)\right]^{-}$.

Define

$$
M=\left(\begin{array}{cccc}
\oplus \bar{A}_{k} & D_{3} & & 0 \\
& \bigoplus A_{k} & D_{1} & \\
& & B & D_{2} \\
0 & & & \bigoplus A_{k}^{a}
\end{array}\right) \text { on } \mathcal{H}_{4} \oplus \mathcal{H}_{1} \oplus \mathcal{H}_{2} \oplus \mathcal{H}_{3},
$$

where $\mathcal{H}_{4}=\oplus \mathcal{H}_{k}^{4}, \bar{A}_{k} \in \mathcal{L}\left(\overline{\mathcal{H}}_{k}\right)(k=1,2, \cdots)$, and

$$
\begin{aligned}
D_{3} & =\left(\begin{array}{lll}
D_{11}^{3} & D_{12}^{3} & \ldots \\
D_{21}^{3} & D_{22}^{3} & \ldots \\
\ldots & \ldots & \ldots
\end{array}\right), \\
D_{k i}^{3} & = \begin{cases}Q\left(\Omega_{2 j}\right), & \text { if } \sigma\left(\bar{A}_{k}\right)=\sigma\left(A_{i}\right)=\bar{\Omega}_{2 j} ; \\
0, & \text { otherwise. }\end{cases}
\end{aligned}
$$

Thus $D_{3}$ is compact.

Suppose that $E \in \mathcal{A}^{\prime}(M)$ is an idempotent and

$$
E=\left(\begin{array}{llll}
E_{11} & E_{12} & E_{13} & E_{14} \\
E_{21} & E_{22} & E_{23} & E_{24} \\
E_{31} & E_{32} & E_{33} & E_{34} \\
E_{41} & E_{42} & E_{43} & E_{44}
\end{array}\right) \begin{aligned}
& \mathcal{H}_{4} \\
& \mathcal{H}_{1} \\
& \mathcal{H}_{2} \\
& \mathcal{H}_{3}
\end{aligned}=\left(\begin{array}{cc}
E_{11} & E_{12}^{\prime} \\
E_{21}^{\prime} & E_{22}^{\prime}
\end{array}\right) \begin{gathered}
\mathcal{H}_{4} \\
\mathcal{H}_{1} \oplus \mathcal{H}_{2} \oplus \mathcal{H}_{3}
\end{gathered}
$$

Then $E_{41}=0$ and $E_{31}=0$, since Lemma 2.1. From Lemma 2.1 and Lemma 2.5, $E_{21}=0$. Since $\sigma\left(\bar{A}_{k}\right)^{0} \cap \sigma\left(\bar{A}_{j}\right)^{0}=\varnothing(k \neq j)$ and since $\bar{A}_{k} \in(\mathrm{SI}), E_{11}=\bigoplus \delta_{k}^{4} I_{k}^{4}$, $\delta_{k}^{4}=0$ or 1 , where $I_{k}^{4}$ is the identity operator on $\mathcal{H}_{k}^{4}(k=1,2, \cdots)$. Since $G \in(\mathrm{SI})$, $E_{22}^{\prime}=0$ or $I_{1} \oplus I_{2} \oplus I_{3}$. Assume that $E_{22}^{\prime}=0$ (if $E_{22}^{\prime}=I_{1} \oplus I_{2} \oplus I_{3}$, consider 
$\left.I_{4} \oplus I_{1} \oplus I_{2} \oplus I_{3}-E\right)$. Thus we have $E_{11} D_{3}+E_{12}\left(\oplus A_{k}\right)=\left(\oplus \bar{A}_{k}\right) E_{12}$. Suppose that

$$
E_{12}=\left(\begin{array}{lll}
L_{11} & L_{12} & \ldots \\
L_{21} & L_{22} & \ldots \\
\ldots & \ldots & \ldots
\end{array}\right),
$$

For each $k$, find $i$ such that $\sigma\left(\bar{A}_{k}\right)=\sigma\left(A_{i}\right)$; then $\bar{A}_{k} L_{k i}-L_{k i} A_{i}=\delta_{k}^{4} D_{k i}^{4}$ implies $\delta_{k}^{4}=0$. Therefore $E=0$ and $M \in(\mathrm{SI})$.

The third step. The recovery of the zero indices.

Take $\lambda_{1 k} \in \partial \bar{\Omega}_{1 k}\left(k=1,2, \cdots, l_{1}\right), \lambda_{3 k} \in \partial \Omega_{3 k}\left(k=1,2, \cdots, l_{2}\right)$. Rearrange

$$
\left\{\lambda_{1 k}\left(k=1,2, \cdots, l_{1}\right), \lambda_{3 k}\left(k=1,2, \cdots, l_{2}\right)\right\}=\left\{\lambda_{k}\right\}_{k=1}^{l_{1}+l_{2}} .
$$

Define $B_{k} \in \mathcal{L}\left(\mathcal{H}_{k}\right)$ by $B_{k} e_{n}=\frac{1}{n k} e_{n-1}(n=2,3, \cdots), B_{k} e_{1}=0$, where $\left\{e_{n}\right\}_{n=1}^{\infty}$ is an ONB of Hilbert space $R_{k}=\mathcal{H}\left(k=1,2, \cdots, l_{1}+l_{2}\right)$. Set $\bar{B}=\bigoplus B_{k} \in \mathcal{L}\left(\mathcal{H}_{5}\right)$, where $\mathcal{H}_{5}=\bigoplus R_{k}$.

Define

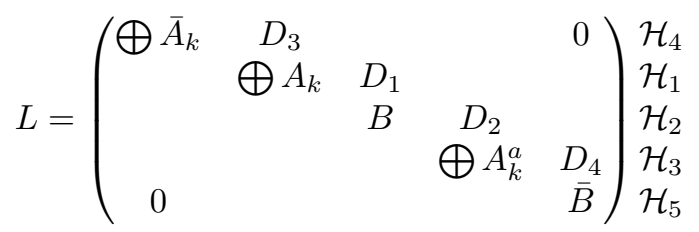

where

$$
D_{4}=\left(\begin{array}{ccc}
D_{11}^{4} & D_{12}^{4} & \ldots \\
D_{21}^{4} & D_{22}^{4} & \ldots \\
\ldots & \ldots & \ldots
\end{array}\right)
$$

and $D_{k j}^{4} \notin \operatorname{ran} \tau_{A_{k}^{a} B_{j}}, D_{k j}^{4}$ is compact if $\sigma\left(A_{k}^{a}\right) \cap \sigma\left(B_{j}\right) \neq \varnothing$ (Lemma 2.6), otherwise $D_{k j}^{4}=0$. Choose $\left\|D_{k j}^{4}\right\|$ so small that $D_{4}$ is compact. By the same arguments used above, $L \in(\mathrm{SI})$.

Set $B_{k}^{\prime}=\lambda_{k}+B_{k} \in \mathcal{L}\left(R_{k}\right)$ and $B^{\prime}=\bigoplus B_{k}^{\prime} \in \mathcal{L}\left(\mathcal{H}_{6}\right)$, where $\mathcal{H}_{6}=\bigoplus R_{k}$.

Define

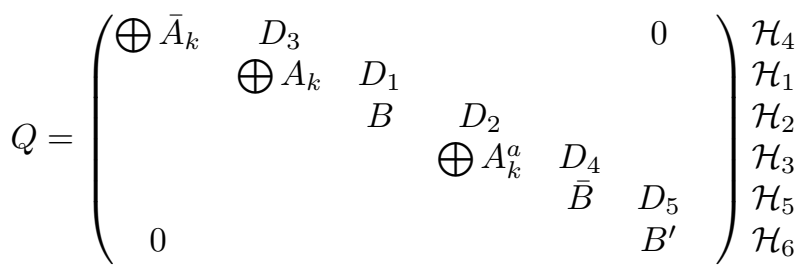

where

$$
D_{5}=\left(\begin{array}{cccc}
D_{1}^{5} & & & 0 \\
& D_{2}^{5} & & \\
0 & & \ddots &
\end{array}\right)
$$

and $D_{k}^{5} \notin \operatorname{ran} \tau_{B_{k} B_{k}^{\prime}}, D_{k}^{5}$ is compact $(k=1,2, \cdots)$ (Lemma 2.7) and choose $\left\|D_{k}^{5}\right\|$ so small that $D_{5}$ is compact. Then $Q \in(\mathrm{SI})$.

Rearrange $\left\{C\left(\Omega_{1 i}\right)\left(i \in \Lambda_{1}\right), C\left(\Omega_{3 k}\right)\left(k \in \Lambda_{3}\right)\right\}=\left\{C_{k}\right\}_{k=1}^{l}$.

Set $C=\bigoplus C_{k}$ on $\mathcal{H}_{7}$, where $H_{7}=\bigoplus_{k=1}^{l} \mathcal{M}_{k}, C_{k} \in \mathcal{L}\left(\mathcal{M}_{k}\right)$; then $\sigma(C)=$ $\left[\bigcup \sigma\left(C_{k}\right)\right]^{-}$. 
Define

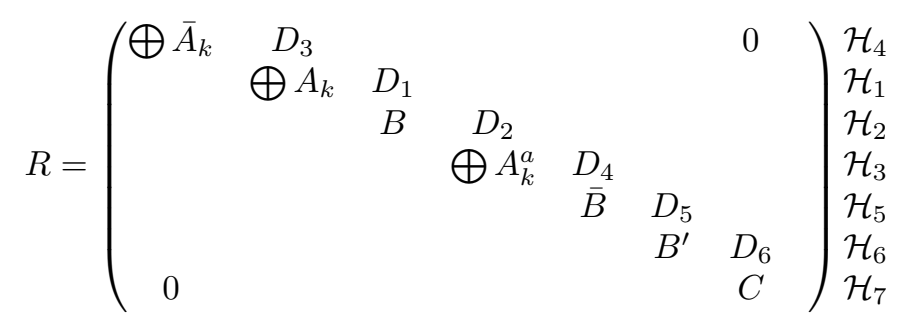

where

$$
D_{6}=\left(\begin{array}{cccc}
D_{1}^{6} & & & 0 \\
& D_{2}^{6} & & \\
0 & & \ddots &
\end{array}\right)
$$

and $D_{k}^{6^{*}} \notin \operatorname{ran} \tau_{C_{k}^{*} B_{K}^{\prime *}}, D_{k}^{6}$ is compact $(k=1,2, \cdots)$ (Lemma 2.7$)$ and choose $\left\|D_{k}^{6}\right\|$ so small that $D_{6}$ is compact. Then $R \in(\mathrm{SI})$.

The fourth step. The recovery of the negative indices.

Assume that $\left\{\bar{C}\left(\Omega_{3 k}\right)\right\}=\left\{\bar{C}_{k}\right\}$ and set $\bar{C}=\bigoplus \bar{C}_{k}$ on $\mathcal{H}_{8}=\bigoplus \mathcal{N}_{k}$ where $\bar{C}_{k} \in \mathcal{L}\left(\mathcal{N}_{k}\right)(k=1,2, \cdots)$; then $\sigma(\bar{C})=\left[\cup \sigma\left(\bar{C}_{k}\right)\right]^{-}$.

Define

$$
S=\left(\begin{array}{ccccccccc}
\oplus \bar{A}_{k} & D_{3} & & & & & & 0 \\
& \bigoplus A_{k} & D_{1} & & & & & & \mathcal{H}_{4} \\
& & B & D_{2} & & & & & \mathcal{H}_{1} \\
& & & \bigoplus A_{k}^{a} & D_{4} & & & & \mathcal{H}_{2} \\
& & & & \bar{B} & D_{5} & & \\
& & & & & B^{\prime} & D_{6} & \\
\mathcal{H}_{5} \\
0 & & & & & & C & D_{7} \\
\mathcal{H}_{6} \\
\mathcal{H}_{7} \\
& & & & & & & \bar{C}
\end{array}\right) \mathcal{H}_{8}
$$

where

$$
D_{7}=\left(\begin{array}{ccc}
D_{11}^{7} & D_{12}^{7} & \ldots \\
D_{21}^{7} & D_{22}^{7} & \ldots \\
\cdots & \ldots & \ldots
\end{array}\right)
$$

and $D_{i k}^{7} \notin \operatorname{ran} \tau_{C_{i}, \bar{C}_{k}}, D_{i k}^{7}$ is compact if $\sigma\left(C_{i}\right)=\sigma\left(\bar{C}_{k}\right)$ (Lemma 2.5), otherwise $D_{i k}^{7}=0$. Choose $\left\|D_{i k}^{7}\right\|$ so small that $D_{7}$ is compact. Then $S \in(\mathrm{SI})$. It is a routine exercise to check that $S=$ essentially normal + compact, $\sigma(T)=\sigma(S), \sigma_{e}(T)=$ $\sigma_{e}(S)$ and $\operatorname{ind}(\lambda-T)=\operatorname{ind}(\lambda-S)\left(\lambda \in \rho_{F}(T)\right)$. Thus by the Brown-DouglasFillmore Theorem [3], there is a compact operator $K$ such that $T+K$ is unitarily equivalent to $S$ and the proof of the Main Theorem is now complete.

\section{REFERENCES}

1. R. A. Adams, Sobolev spaces, Academic Press, New York-San Francisco-London, 1975. MR 56:9247

2. C. Apostol, L. A. Fialkow, D. A. Herrero and D. Voiculescu, Approximation of Hilbert space operators, II, Research Notes in Math., vol. 102, Pitman Books, Ltd., London-Boston-Melbourne, 1984. MR 85m:47002 
3. L. G. Brown, R. G. Douglas, and P. A. Fillmore, Unitary equivalence modulo the compact operators and extensions of $C^{*}$-algebras, Proceedings of a Conference on Operator Theory, Halifax, Nova Scotia, 1973, Lect. Notes in Math., vol. 345, Springer-Verlag, 1973, pp. 58-128. MR 52:1378

4. J. B. Conway, Subnormal operators, Research Notes in Math. 51 (1981). MR 83i:47030

5. M. Cowen and R. G. Douglas, Complex geometry and operator theory, Bull. Amer. Math. Soc. 83 (1977), 131-133. MR 58:17885

6. M. Cowen and R. G. Douglas, Complex geometry and operator theory, Acta Math. 141 (1978), 187-261. MR 80f:47012

7. R. G. Douglas, Banach algebra techniques in operator theory, Academic Press, New York and London, 1972. MR 50:14335

8. C. K. Fong and C. L. Jiang, Approximation by Jordan type operators, Houston J. Math. 19 (1993), 51-62. MR 94d:47011

9. C. K. Fong and C. L. Jiang, On irreducible operators, Northeastern Math. J. 8 (5) (1992), 385-390. MR 94b:47023

10. F. Gilfeather, Strong reducibility of operators, Indiana Univ. Math. J. 22 (1972), 393-397. MR 46:2460

11. P. R. Halmos, Irreducible operators, Mich. Math. J. 15 (1968). MR 37:6788

12. D. A. Herrero, Approximation of Hilbert space operators, Vol. 1, 2nd ed., Pitman Res. Notes Math. 224, Longman Sci. Tech., Harlow, Essex, 1989. MR 91k:47002

13. D. A. Herrero and C. L. Jiang, Limits of strongly irreducible operators and the Riesz decomposition theorem, Mich. Math. J. 37 (1990), 283-291. MR 91k:47035

14. D. A. Herrero, T. J. Taylor and Z. Y. Wang, Variation of the point spectrum under compact perturbations, Topics in operator theory, Constantin Apostol Memorial Issue, OT: Advances and Applications, vol. 32, Birkhäuser-Verlag, Basel-Boston-Stuttgart, 1988, pp. 113-158. MR 89h:47018

15. Z. J. Jiang, A lecture on operator theory, The report in the seminar of functional analysis in Jilin Univ., Chang Chun, 1979.

16. Z. J. Jiang and S. L. Sun, On completely irreducible operators, Acta Scientiarum Naturalium Universitatis Jilinensis 4 (1992), 20-29.

17. C. L. Jiang, Strongly irreducible operators and Cowen-Douglas operators, Northeastern Math. J. 7 (1) (1991), 1-3. MR 92e:47024

18. C. L. Jiang, Similarity, reducibility and approximation of Cowen-Douglas operators, J. Operator Theory 32 (1994), 77-89. MR 96d:47013

19. C. L. Jiang and Z. Y. Wang, The spectral pictures of completely irreducible operators and decomposition theorem of Hilbert space operators, Northeastern Math. J. 10 (2) (1994), 145148.

20. C. L. Jiang and Z. Y. Wang, A class of strongly irreducible operators with nice property, J. Operator Theory (to appear).

Department of Mathematics, Jilin University, Changchun 130023, P.R. of China

Department of Mathematics, Sichuan University, Chengdu 610064, P.R. of China

Department of Mathematics, East China University of Science and Technology, Shanghai, 200237, P.R. of China 